\title{
Assigning Functions to Medical Technologies
}

\author{
Alexander Mebius ${ }^{1,2,3}$ (D)
}

Received: 5 January 2016 / Accepted: 3 October 2016 / Published online: 14 November 2016 (C) The Author(s) 2016. This article is published with open access at Springerlink.com

\begin{abstract}
Modern health care relies extensively on the use of technologies for assessing and treating patients, so it is important to be certain that health care technologies (i.e., pharmaceuticals, devices, procedures, and organizational systems) perform their professed functions in an effective and safe manner. Philosophers of technology have developed methods to assign and evaluate the functions of technological products, the major elements of which are described in the ICE theory. This paper questions whether the standard of evidence advocated by the ICE theory is adequate for ascribing and assessing technologies employed in health care. The paper proposes that the general problem with the standard of evidence embodied in the ICE theory (i.e., testimony and evidence of mechanisms) is too permissive for assessing medical technologies, in that it does not take into account the relative benefit and harm of medical technologies in ensuring safe functional performance in patients. The paper illustrates how evidence-based medicine (EBM) has demonstrated the value of clinical research methods, including observational studies, randomized and non-randomized clinical trials, and formal techniques, such as meta-analysis, to measure therapeutic effectiveness. I argue, therefore, that evidence from clinical research studies should take precedence over the testimonial evidence and other types of non-clinical evidence, in providing justification for health technologies.
\end{abstract}

Keywords ICE theory · Health technologies · Theory of mechanisms · Technological evidence $\cdot$ Functional ascription $\cdot$ Evidence-based medicine

Alexander Mebius

alexander.mebius@kellogg.ox.ac.uk

1 Nuffield Department of Primary Care Health Science, University of Oxford, Radcliffe Primary Care Building, Radcliffe Observatory Quarter, Woodstock Road, OX2 6GG Oxford, UK

2 Munich Center for Mathematical Philosophy, Ludwig-Maximilians-Universität (LMU), München Geschwister-Scholl-Platz 1, D-80539 München, Germany

3 Department of Philosophy and History of Technology, Royal Institute of Technology (KTH), Teknikringen 78 B, 10044 Stockholm, Sweden 


\section{Introduction}

Health technologies play a crucial role in medicine, and modern health care relies extensively on the use of technologies for assessing and treating patients. As in most practical endeavors, the action-oriented nature of medical technologies entails their frequent use for attaining health-related goals and outcomes in treating patients and managing disease. For this reason, it is of interest to patients, clinicians, and policy makers that these technologies can be relied upon to perform their professed functions in an effective and safe manner. Although the impact of technological use on health care practice cannot be overstated, it often remains unclear whether medical functions have been assigned properly, and what is required to distinguish useful from useless, or even harmful, technologies.

Philosophers of technology have devoted substantial effort to analyze what it means to assign functions to technological products. One important account of functional ascriptions is the ICE theory of functions (Houkes et al. 2010, 2011; Vermaas and Houkes 2006). The ICE analysis attempts to characterize technological artifacts as intentionally designed products with functions that are utilized by agents for attaining goals by following a set of actions. This set of actions is specified by a use plan, which relates information about the proper use and function of these technologies. For the use plan to be valid, it must be justified on the basis of some relevant evidence. Relevant evidential support that the ICE theory identifies for this purpose includes knowledge about physiochemical capacities (i.e., biotechnological mechanisms), individual experience, expertise, and testimony. Although ICE theorists commonly enumerate several possible sources of evidence, testimonial evidence and physiochemical reasoning about biotechnological processes or mechanisms have received specific epistemological attention for attaining functional knowledge. For example, the ICE theorists are often explicit about taking "testimony seriously as a source of evidence" (Houkes 2006, p. 107). As suggested by Vermaas and Houkes (2006), "Testimony is not just a basis for the effectiveness, capacity and contribution beliefs that are involved in function ascriptions, but also provides a basis for this privileging" (p. 114).

Note that ICE theorist is not committed to merely describing how action-oriented technologies (e.g., health care products) are commissioned and used in different contexts. Rather, proponents of the ICE theory of evidence seek to provide a normative framework for evaluating and justifying technological use. As Houkes and Vermaas (2010) put it, "we approach both artefacts and the actions in which they play a role largely from a normative rather than a descriptive perspective. We do not offer a theory about how people actually use or design artefacts, or how they in fact describe them in functional terms; instead we seek to provide a framework for evaluating some aspects of these activities, and we theorise about rational and proper artefact use, and about justifiable function ascriptions" (p. 4).

This paper contends, however, that the evidential framework that the ICE theorists endorse proves inadequate for assigning functions to technologies intended for medical purposes. It is argued that ICE functions are not justified as an appropriate evidential standard that should be required for justifiably 
assigning functions to health care technologies. ${ }^{1}$ I suggest that ICE theory's endorsement of testimony as serious source for justification entails an inadequate theory of evidence for basing functional ascriptions to medical technologies. ${ }^{2}$ This paper also argues that the evidential role ascribed to mechanisms (i.e., information about physiochemical capacities from basic biomedical research) has been exaggerated, emphasizing more balanced appraisals of the usefulness of mechanisms with respect to their epistemic value in the medical sciences and biotechnological innovation. Promising as its application may appear for biotechnological fields such as clinical pharmacology and drug development, the evidential value of mechanisms has not been empirically shown to be successful in these areas (Mebius et al. 2016). Translating evidence from laboratory research on mechanisms and/or relying on clinical testimony risks drawing a biased and incomplete picture of the functional merits of medical technologies that can lead to the adoption of ineffective or even harmful treatments.

Section 2 presents the Vermaas and Houkes ICE theory for analyzing technological artifacts and discusses its relevance for assigning functions to medical technologies. Section 3 argues that the ICE account provides a theory of evidence that is unacceptable for the assessment of health technologies. In section 4, I show that faulty or inappropriate evidence risks misleading functional ascriptions and argue that few, if any, of the sources of evidence enumerated by the theory meets the desired evidential standard for reliably assigning functions to technologies for clinical purposes. Section 5 proposes that ascribing functions to technologies in health care can only be justified by evidence of high quality from comparative clinical studies. Section 6 clarifies what high-quality evidence entails, and what it means for a health care technology to "function" in a clinically relevant sense. This is done by placing the further constraint that the type of justification needed for ascribing medical functions to artifacts should require evidence that technologies are clinically effective meaning that their use entails (1) patientrelevant benefits that outweigh any harm, (2) that they are clinically applicable to their intended target population of patients, and (3) they are the best available option for the clinical purpose.

\section{The ICE Theory of Functions}

A general way to think about medical technologies is that they are material objects developed for use in some form of human activity. The roles played by technologies in these activities are in some theoretical accounts considered to be more or less the product of intentional and social elements, as opposed to being determined by the physiochemical capacities inherent in the technologies themselves (Bijker 1987). Meanwhile, other theorists of technological functions maintain that institutional and

\footnotetext{
${ }^{1}$ Although my aim here is to consider functions ascribed to health technologies, the discussion might also be relevant to the analysis of technological use in non-clinical contexts, for example, in assessing technological use related to social policy interventions (c.f. La Caze and Colyvan 2016).

${ }^{2}$ I follow the Health Technology Assessment Programme (HTA) in defining "health technology" or "medical technology" as: "An intervention that may be used to promote health, to prevent, diagnose or treat acute or chronic disease, or for rehabilitation. Health technologies include pharmaceuticals, devices, procedures and organizational systems used in health care" [http://htaglossary.net/tiki-index.php?page=health].
} 
social backgrounds are interconnected with physical structures in determining the functional status of technological artifacts (Kroes 2002; Houkes et al. 2011).

Vermeer and Houkes' ICE theory is one of the most prominent accounts of technological functions and an example of the interconnected approach. The ICE theory of functions, first introduced by Vermaas and Houkes (2003) and Vermaas and Houkes (2004; Houkes and Vermaas 2010), provides an analysis of functional ascriptions to technologies that reads as follows:

"An agent $a$ ascribes the capacity to $\phi$ as a function to an artefact $\mathrm{x}$, relative to a use plan $p$ for $\mathrm{x}$ and relative to an account $A$, if:

I. the agent $a$ has the capacity belief that $\mathrm{x}$ has the capacity to $\phi$, when manipulated in the execution of $p$, and the agent a has the contribution belief that if this execution of $p$ leads successfully to its goals, this success is due, in part, to x's capacity to $\phi$;

C. the agent can justify these two beliefs on the basis of $A$; and

E. the agents $d$ who developed $p$ have intentionally selected $\mathrm{x}$ for the capacity to $\phi$ and have intentionally communicated $p$ to other agents $u$." (Vermaas \&and Houkes 2006, p. 9)

The I condition maintains that the agent ascribing the function should believe that the functional capacity materializes when the technology is implemented in accordance to the use plan. Condition $\mathrm{C}$ requires that the agent ascribing the function must be able to justify his or her belief that the artifact will perform its intended function according to a pre-specified use plan. Importantly, condition $\mathrm{C}$ requires more than that the agent who makes an ascription believes that the artifact has a functional capacity (as required by condition I). What it requires in addition is that the agent cites a relevant source of evidence that justifies his or hers belief that the artifact is functionally effective. For this purpose, the ICE theory of evidence considers the following evidential sources:

"The relevant evidence may be experience that the artefact has the capacity, testimony by other agents, or scientific or technological knowledge; in all cases, the evidence supports the function ascription by supporting the beliefs that the artefact has the corresponding physicochemical capacity and that this capacity explains, in part, the effectiveness of a use plan." (Houkes and Vermaas 2010, p. 102)

Although numerous sources of possible evidential justifications are cited by the authors in this passage and elsewhere, including knowledge of physiochemical capacities, experience of effective performance, and testimonies from other users or designers, evidential support can generally be provided by "any agent who develops and communicates a use plan and who can justify it, if only by plain experience that it works" (Vermaas and Houkes 2006, p. 9).

ICE theorists plausibly assume that an ascription of function needs and contains a justification. A manufacturer of a drug or diagnostic agent is normally required to justify the claimed performance of its product by citing relevant evidence for its 
functional effectiveness. This allows claims about the efficacy and safety of technologies to be challenged on evidential grounds. However, it is a mistake to suppose, or so I shall argue, that testimonial evidence along with other ICE evidential types provides an adequate evidential standard for justifying that medical technologies are able to function properly.

A general problem with the ICE condition $\mathrm{C}$ is that it is too permissive in the sort of evidence that it allows to be cited in support of I. This is also noted by Hansson (2006), who similarly observes that such a general theory of evidence entails the unwanted consequence that "the condition $\mathrm{C}$ will almost always be satisfied when the I condition is satisfied" (p. 20). One might suggest resolving the problem by modifying condition $\mathrm{C}$ as to exclude weaker forms of evidence, such as testimony, as justifiers of functional ascriptions. However, this proposal seems to be at odds with the subject-dependent notion of function on which ICE justifications are based: "In the case of technical functions, we have both the subject-independent notion $\mathrm{x}$ has the function $\phi$ ' and subject-dependent notions such as $a$ believes that $\mathrm{x}$ has the function to $\phi$ '. Vermaas and Houkes have chosen to define a subject-dependent notion, namely that of a single individual's ascriptions of a function to an object." (Hansson 2006, p. 20). Moreover, excluding subject-dependent forms of evidence, such as testimony, would be inconsolable with a theory of evidence that maintains testimonial evidence is indispensable: "Testimony is not just a basis for the effectiveness, capacity and contribution beliefs that are involved in function ascriptions, but also provides a basis for this privileging" (Vermaas and Houkes 2006, p. 114).

In any case, the ICE theory arguably employs too broad a concept of evidence for assigning and evaluating medical functions. The most significant issue with this notion of evidence is its reliance on testimony and preclinical information about biotechnological mechanisms, which puts it at odds with the evidential standards found in clinical research and practice. In the following section, I discuss why the ICE theory of evidence proves inadequate for circumscribing functions in the case of health care technology. I also show why the type of evidence enumerated by its proponents, such as testimony and/or theories and models of physicochemical capacities (biotechnological mechanisms), is inappropriate for reliably describing health technologies effectiveness and safety.

In the following section, it is argued that the ICE epistemology of functional ascription proves inadequate for assigning clinically relevant functions to medical technologies because of the very high risk of bias generally inherent in expert testimony and evidence of mechanisms. I show that expert testimony is problematic because some experts may claim that a technological intervention is effective while other experts may disagree. The key question then is how to determine the relative value of a biotechnological intervention given contradictory claims about the safety and effectiveness of that intervention.

\section{Testimony and Biotechnological Mechanisms as Evidence}

Philosophers of technology have insisted that expert testimony and evidence of biotechnological mechanisms can be used to support the assigning of functions to medical technologies. Yet, evidence of mechanisms does not feature in dominant EBM 
standards of evidence. This section examines evidential standards in medicine and suggests why evidence, such as testimony and mechanistic reasoning, has ceased to play a significant role in supporting functional ascriptions to health technologies.

Testimony is a form of evidence based on personal experience that is often invoked in establishing consensus among experts. Evidence-based medicine rose to prominence after a long predominance of consensus conferences for assessment of medical technologies. A couple of years before evidence-based medicine (EBM) methodology was adopted throughout health care for guiding health research and patient care, Mario Bunge stated, in his book on the philosophy of technology, "Medicine...is now on the right track, though it has a long way to go before attaining the rigor and effectiveness of engineering" (1985, p. 246). At that time, the evaluation of medical technologies was still being dominated by pre-EBM conceptions of clinically reliable evidence, such as “expert consensus." As late as 1990, governmental agencies and health care regulators responsible for assessing the use of technologies relied on testimony and personal experience as indispensable sources of evidence:

"Group judgment is perhaps the most widely used means of assessment of medical technologies in many countries. The consensus development conference is a relatively inexpensive and rapid mechanism for the consideration and evaluation of different attributes of a medical technology including, for example, safety, efficacy, and efficiency, among many others." (Baratz and Goodman 1990)

Only a few years later, after the introduction of the EBM in medicine, expert consensus was viewed by the same regulatory agencies and health experts as the lowest level of evidence. For example, the Canadian Task force on Preventive Health Care stated that "Evidence takes precedence over consensus" (Canadian Task Force on Preventive Health Care). From its introduction just over two decades ago, EBM has come to dominate medical practice, teaching, and policy. EBM initially defined itself as a method that.

"de-emphasizes intuition, unsystematic clinical experience, and pathophysiological rationale as sufficient grounds for clinical decision making and stresses the examination of evidence from clinical research." (Evidence-Based Medicine Working Group 1992)

Although the original definition has been somewhat refined (partially as the result of legitimate critique of its methodology ${ }^{3}$ ), EBM research has continued to dominate the field of health care. EBM rules of evidence are, for instance, employed by policy makers in directing clinical guidelines (Howick et al. 2015), and EBM research methods are endorsed by the editorial policies of leading medical journals, such as the Journal of the American Medical Association (JAMA) and the British Medical Journal (BMJ). Some of the most crucial aspects of the EBM theory of evidence lies in

\footnotetext{
3 The subsequent definition emphasizes the role of values: "Evidence-based practice is the integration of clinical expertise, patient values, and the best research evidence into the decision making process for patient care. Clinical expertise refers to the clinician's cumulated experience, education and clinical skills. The patient brings to the encounter his or her own personal preferences and unique concerns, expectations, and values. The best research evidence is usually found in clinically relevant research that has been conducted using sound methodology" (Sackett 1996, p. 71)". Other criticisms of EBM have pointed out a range of issues, such as an overemphasis on randomization and clinical trials (Worrall 2002).
} 
its emphasis on the use of systematic reviews and the superiority of comparative clinical studies (including observational studies and randomized trials) over pathophysiologic rationale (descriptions of biological and biotechnological mechanisms) and expert judgment. EBM provides a set of formal methods and techniques for evaluating the effectiveness of medical technologies. The techniques implemented by EBM are especially useful for evaluating treatments that present incremental advantages to current treatment standards. EBM methods have been extended in recent years to areas such as public health interventions, which offer promising ways of testing, for example, technologies outside clinical settings (e.g., food policy programs) (but c.f. Cartwright and Hardie 2012).

Ascribing the use of health technologies on the basis of weak evidence supporting claims to functional effectiveness and safety arguably puts patients at risk because questions about their harm and benefit are inadequately addressed. One glaring example from the history of medicine is the use of the lancet. This instrument was used primarily for bloodletting patients suffering from various conditions. Until the mid-19th century, most physicians believed that the lancet afforded a far superior method for treating various diseases. Most physicians accordingly ascribed this function to the lancet. The Edinburg physician William Buchan, for example, claimed in his influential 1769 medical guide Domestic Medicine that:

"No operation of surgery is so frequently necessary as bleeding...Bleedings for topical inflammations ought always to be performed as near the part affected as possible. When this can be done with a lancet, it is to be preferred to any other method; but where a vein cannot be found, recourse must be had to leeches or cupping. The quantity of blood must be always be regulated by strength, age, constitution, manner of life, and other circumstances relating to the patient. It would be ridiculous to suppose that a child could bear to lose as much blood as a grown person, or that a delicate lady should be bled to the same extent as a robust man." (Buchan et al. 2012, p. 30)

In a somewhat similar vein, the essentiality of bloodletting was expressed by Thomas Smith in his Extracts from the Diary of a Huntsman from 1838: “...the best thing [a huntsman] can do, if [his] horse is distressed, is to have about two or three quarts of blood taken from him, see that he has some gruel and is properly taken care of...” (Smith 1921, p. 65).

During its popularity, many explanations were put forward by some of the most eminent physicians to explain the alleged effectiveness of therapies such as bloodletting, vomiting, blistering, and purging by referencing contemporary authorities and Galenic theories along "humoral" lines that mostly proved to be harmful to the patient (Tröhler 2012). Similarly, many biotechnological innovations will have promising medical functions that often find support in animal models that are well-studied, corroborated theories, and various laboratory findings. It is often the case, however, that one finds technologies with well-supported preclinical evidence to be non-functional or insufficiently effective when it comes to producing the expected benefit in clinical settings (Pound and Bliss 2014). This is why researchers continuously point out that expert testimony, similar to 
laboratory research, provides very limited justification when it comes to reliably establishing the efficacy and safety of medical procedures. This issue is often commented upon by medical researchers, as seen here by Dave Sackett, one of the founders of the EBM movement:

"Millions of dollars' worth of bench research that appeared to show that a reduction in atherosclerosis-related oxidative stress with vitamin $\mathrm{E}$ therapy was recently tested in an RCT[Randomized Controlled Trial] that asked the vital question: Does vitamin E therapy endorsed by this research really help prevent heart disease? The answer was a resounding 'No'." (Sackett et al. 1999, p. 1414)

To take another example, consider the functional justification of vertebroplasty. Vertebroplasty is a medical technique developed for alleviating pain associated with bone fracture. This technique consists of injecting polymethylmethacrylate cement into the fractured bone and was introduced during the mid-1990s. Numerous researchers began reporting remarkable effects of this procedure (Jensen et al. 1997), and vertebroplasty was reimbursed by Medicare in 2001, even though no proper comparative clinical studies were performed to establish that the procedure was functionally safe and effective (Prasad and Ho 2014). In 2004, 24,000 vertebroplasties were reported to have been performed in the United States alone. During this period of widespread implementation, several mechanistic explanations were put forward that supposedly explained the physiochemical properties underlying vertebroplasty's effective use: "the stabilization of the fractured vertebrae; reduction of mechanical stress; and destruction of the nerve endings because of the cement's mechanical, chemical, cytotoxic, and thermal activity as well as its anti-inflammatory action" (Chen et al. 2004, p. 229).

In 2009, however, results from two independent comparative clinical trials were published in the New England Journal of Medicine which showed that the procedure was no more effective than sham procedures (Buchbinder et al. 2009; Kallmes et al. 2009). This example of vertebroplasty and Sackett's example of the ineffectiveness of vitamin E therapy illustrate the potentially harmful or wasteful practice of assigning functions to technologies by translating preclinical effectiveness beliefs, or mechanistic theories about purported functions, that consequently fail to materialize. In evaluating technologies for treatment purposes, there are usually many uncertainties and concerns about effectiveness, and if, in fact, an intervention leads to more harm than good. One clear epistemic virtue of, for example, the methodology inherent in a properly conducted randomized trial is that it decreased the level of uncertainty by reducing the likelihood of bias and the role of chance interfering with the results (Evans et al. 2010).

I do not contend that some types of technological knowledge relate to conventions that are generally agreed upon for different purposes. For example, knowing that the insulation of the ground wire should be striped in yellow and green (according to standard convention) is a highly useful piece of technological knowledge for an electrician. It might be said that "the experienced doctor can often make a correct diagnosis within his area of expertise without doing a full examination...," and that knowledge of this type "can only be learned through experience" (Nordström 2011, p. 
8). However, the fact that doctors make the correct diagnosis based on their expertise is often not enough.

To take an example, research reports of diagnostic studies suggest that the clinical accuracy of malignant melanoma ranges from 64 to $80 \%$ (Marghoob et al. 2009). This means that up to one third of all patients presenting with melanomas are likely to be misdiagnosed even after a full examination. Although it might be possible that an experienced doctor will be able to achieve a high degree of accuracy, few would contend that their diagnostic ability (expert judgment) is sufficiently adequate for detecting melanoma, given that even the most seasoned dermatologist $(\geq 10$-year experience) can, at his best, only achieve an $80 \%$ success rate (Morton and Mackie 1998). This, in fact, explains why the misdiagnosis of this type of cancer is among the most commonly cited causes for malpractice litigation initiated against clinicians. Of course, achieving a $100 \%$ success rate is not a feasible goal in many cases. My only point here is that clinical experience, like many forms of expertise, is often too fallible a source of evidence.

I hope that this section has explained why functional ascriptions based on weaker forms of evidence, such as testimony, expert judgment, and physiochemical (mechanistic) reasoning (or combinations of these), provide inadequate evidential support. The next section argues that well-performed comparative clinical studies are indispensable in guiding functional ascriptions of medical technologies. These types of studies have, for example, the distinctive advantage of being able to capture small to moderate improvements in health-related outcomes following the use of a technology. That is, clinical studies are able to measure and compare continuous variables, and this can establish the degree of functionality of a technology.

\section{Evidence-Based Clinical Studies}

In this section, we consider how evidence from comparative clinical research can inform and constrain the ascription of functions to medical technologies. I argue that evidence from clinical studies takes precedence over other types of evidence concerning the functionality of health technologies because clinical studies provide strong justification for medical interventions by, for example, providing a specific estimate of functional efficiency and effectiveness. That is, they often provide a continuous estimate, rather than a categorical estimate, of therapeutic effectiveness, and they have the ability to compare such estimates to find out which one is more beneficial. ${ }^{4}$ As such, measures obtained from comparative clinical studies often permit the quantification of the strength of effectiveness associated with a particular dose in a particular setting in a particular population.

The methodological importance of comparative clinical studies can partly be inferred from their widely adopted use for evaluating technological functions (Guyatt et al. 2008; Higgins and Green 2011; Straus et al. 2011). Clinical research methods often applied for such purposes include observational studies (e.g., retrospective and

\footnotetext{
${ }^{4}$ I use the term "clinical studies" to mean experimental (randomized or non-randomized) clinical trials and observational (or non-experimental) studies.
} 
prospective cohort studies), randomized or non-randomized clinical trials, and different formal techniques, such as the meta-analysis of data to synthesize evidence obtained from clinical studies. The evidence generated by these methods is considered to be more reliable than, for example, laboratory research and expert testimony, because evidence from clinical studies is less likely to suffer from systematic bias (OCEBM Levels of Evidence Working Group 2011, Schünemann et al. 2008).

Comparative clinical trials, for example, have the advantage of being able to employ measures to protect against confounding factors, such as random allocation of subjects, concealment of treatment conditions, and double blinding (Howick \& Mebius 2014; Mebius 2015), which methodological research can significantly reduce the risk of systematic bias (Schulz et al. 1995; Howick 2016). Additional methodological criteria for assessing the quality of synthesized data from clinical studies are usually put in place to further minimize the risk of bias (see, for example, Shea et al. 2007). In short, from the perspective of clinical research and practice to assess the validity of technological functions, clinical studies are better equipped to protect the assessment of functionality from possible confounding variables that might distort the evaluation process.

Comparative clinical studies also have the advantage that they can estimate the effectiveness of a technological intervention relative to a comparable technological intervention in humans. Comparative clinical studies, such as randomized controlled trials, are thus able to estimate the degree of efficacy that a technological intervention is able to provide. Such information becomes advantageous when, for example, assessing how much an intervention is able to reduce pain and improve function in patients with osteoarthritis of the knee (Fransen and McConnell 2008). This further allows a comparison to be made between health technologies targeting similar treatment pathways. For example, a study might show that less invasive techniques (such as ultrasonography) may be equal to or diagnostically better than more invasive techniques, such as angiography (Knottnerus et al. 2009).

No corresponding method seems to exist in reasoning about physiochemical structures (mechanisms), or arriving at a decision by consensus, which allows the same systematic and precise estimates of the comparative efficacy (or inefficacy) of medical technologies, which can detect seemingly small (yet clinical significant) differences in functionality. Another advantage of comparative studies is that they are informed by data gathered from implementing technologies in actual clinical settings (unlike experiments on animals under laboratory conditions). That is why most guidelines and national medical agencies require beliefs about functional efficiency and safety to be grounded in evidence from clinical studies, such as observational studies, randomized controlled trials, or systematic reviews and meta-analyses of high-quality evidence obtained by these research methods.

Proponents of the ICE theory and/or mechanistic research might object by maintaining that medical functions are different from other types of technological functions. However, because much technological development directly and indirectly involves developing tools for managing, restoring, or repairing some aspect of human health, a large proportion of technology proper would then have to be abandoned by the ICE theory of technological functions. This would exclude the ascription of functions to synthesized drugs (e.g., aspirin), biotechnologies (e.g., 
viral antigen tests), and technology proper (e.g., MRI, functional MRI, CT, PET, and many others). For example, the Health Technology Assessment Programme (HTA) (which is part of the British National Institute of Health Research) provides the following description:

“"Technologies' in this context mean any method used to promote health; prevent and treat disease; and improve rehabilitation or long-term care. They are not confined to new drugs and include any intervention used in the treatment, prevention or diagnosis of disease." (HTA 2014)

As previously noted, the aim of regulatory bodies and governmental agencies, such as HTA, is to promote evidence-based research methods for establishing the comparative effectiveness and safety of medical technologies. In the case of HTA, evidence of investigated technologies (new or preexisting) is meant to provide justification for their use within the National Health Service (NHS). Correspondingly, the National Institute of Health and Clinical Excellence (NICE) bases its policies and guidelines on the results of EBM research. Moreover, because evaluations of policy interventions are increasingly utilizing the evidential standards originally developed by the EBM movement, it is likely that the proponents of ICE functions will need to abandon it as a theory of evidence for many social policy implementation of technologies, as well (see, e.g., Vodopivec-Jamsek et al. 2012).

Although the requirements for functional ascriptions, today, have been substantially improved, a great deal of functional ascriptions is still based on weak types of evidence. The next section discusses the conditions under which a medical technology should be considered to have a clinically effective function. These considerations are then applied in the formulation of evidential criteria required for assigning functions to technological artifacts intended for health care purposes.

\section{Clinically Effective Technological Products}

The section suggests the type of restrictions on evidential warrants required to adequately determine clinical impact in terms of effectiveness, relevance, and safety. It should be apparent that better evidence allows researchers, health care providers, and policy makers to evaluate the effectiveness of different health interventions better, and that more efficient evidential standards allow for more ethical and resourceful health care decisions about medical technologies.

To begin, it should be noted that it is not necessary to understand the details regarding the causal interactions between biological and technological mechanisms. Often the function, safety, and efficacy of a device, drug, or diagnostic tool will be well documented even though knowledge about the physiochemical properties of the biological and technological mechanisms will be severely lacking or otherwise incomplete. There are many examples where the findings from comparative clinical studies would overturn earlier medical technologies that were adopted on the basis of weak evidence. It has been argued that many of these ascriptions of functional effectiveness overlooked available evidence from comparative clinical studies that indicated these interventions were ineffective (i.e., they did not serve their ostensible function) and/or harmful (Prasad et al. 2014). 
For example, mechanistic evidence suggesting that monoclonal antibodies perform well in critically ill infected patients have been found to be ineffective by clinical research (McCloskey et al. 1994). Another well-known example is the Cardiac Arrhythmia Suppression Trial (CAST) which overturned the advice from expert testimony that treating patients with encainide and flecainide after a myocardial infarction would reduce mortality, when instead, it significantly increased mortality (and according to some estimates resulted in more premature deaths in the USA than the number of US soldiers killed in action during the Vietnam War) (Moore 1995). Finally, the deleterious advice given by Dr. Spock in his bestselling book Baby and Child to place babies on their stomach to sleep led to tens of thousands of avoidable crib deaths (Gilbert et al. 2005). Hence, clinical research not only has a better grasp of function than structure, but it can also correct misplaced assumptions about physiochemical capacities of mechanisms.

As suggested earlier, the EBM methodology maintains that evidence about treatment effects of medical technologies is most reliably obtained from tightly controlled clinical studies. This view is partially based on the notion that the methods used in these studies can be characterized as directly action guiding. For example, Hansson (2014) suggests that clinical trials, one of the key study designs in EBM, represent the "gold standard" of justification in medicine because they can provide evidence that directly guides action: "Clinical trials are directly action-guiding experiments on treatment effects" (2014, p. 41). In his historical survey, Hansson compares the utility of the modern clinical experimental tradition, with its utility to directly guide action, to (technological) experiments conducted by craftspeople in pre-scientific times, concluding that evidence from high-quality clinical trials provides a "gold-standard" of evidence compared with non-clinical studies (see also Hansson 2015).

Having considered the evidence that clinical studies provide the most appropriate type of justification for assigning functions to medical technologies, we now turn to the question of how to distinguish between relevant and high-quality evidence of this type. The decision to implement technologies in health care practice necessarily requires evidence that these technologies are clinically effective. The idea of what is meant by clinically effective is nicely conveyed by Howick (2011): "clinically effective....means that the treatment has (1) patient-relevant benefits that outweigh any harms, (2) is applicable to the patient being treated, and (3) is the best available option" (p. 24). Arguably, it seems clear that technologies that do more harm than good should not be described as having a function in the "effective" sense of the word. As Ashcroft remarks in a paper titled "What is clinical effectiveness?": "From a prescriber's point of view, and from a consumer's, it would be a misuse of language to say that a drug whose side-effects are such that they outweigh its benefits is effective" (2002, p 226). ${ }^{5}$

Although establishing the effectiveness of a technology is a necessary condition for its implementation in clinical practice, it is not a sufficient condition. To assess the effectiveness of a health care technology, we first need to specify the treatment context in which its medical function might be relevant. In addition to being adequately

\footnotetext{
5 This is not the place to debate ethical concerns that may arise from the inadvertent use of technological products. However, it is important to stress that the ethically viable use of medical technologies would undoubtedly require an adequate assessment and evaluation of functionality. Therefore, one might consider it essential to stress the importance of reliable evidence in ethical considerations about technological use in healthcare (Chalmers \& Glasziou 2009).
} 
effective and safe, the technology has to be at least equally effective to any competing technology with an established function for the same purpose in the same treatment situation. Hence, an assessment of medical products for human use must prove their relative effectiveness (Parvizi and Woods 2014), and the technology to be used should be assigned a function for that health care purpose. The less effective technology will either be abandoned or serve as a backup should the primary option fail.

Of course, it is often the case that two technologies can be used to perform the same functional task. However, it is very rare to find two technologies performing a medical function with the same level of effectiveness or efficacy. In all likelihood, the efficacy by which functions are performed will vary within a range of functional efficacy in which different technologies operate. This enables researchers to determine the relative utility of technologies in accordance with their functional performance on specific medical tasks. This is helpful, for example, in deciding the relevant dose in a specific treatment context, by specifying the dosage interval and duration of treatment.

Determining the ability of health technologies to perform for a particular health care function requires sorting clinical functions according to their effectiveness and safety. This is because a medical technology can be more or less (clinically) effective than comparable alternative technologies intended for the same functional task. One might consider, for instance, the kits routinely used in virology diagnostics. Several techniques have been devised for detecting viruses, including the use of electron microscopy to detect viruses from clinical specimens (for example, to observe individual virus particles), viral antigen detection, light microscopy (to visualize and map bacteria infected by viruses), and many more. Additionally, viral tests can be used to monitor the effectiveness of vaccination. Many of these kits have been commercially designed to detect or identify the same bacterial pathogens and to diagnose the same viral infections. In other cases, the same kit is easily modified to serve the purpose of diagnosing different viral infections. For this reason, it becomes important to take into account that the effectiveness and accuracy for detecting and identifying viruses can vary significantly between individual kits or functions and that some tests are highly effective, whereas others are moderately or almost completely ineffective.

A similar example can be made with synthesized substances. For example, evidence suggests that aspirin administered daily in low doses helps to prevent stroke, whereas the same drug in higher doses (several tablets per day) is no more effective in preventing stroke, but entails an increased risk of stomach ulcers (Antithrombotic Trialists' (ATT) Collaboration 2009). A functional ascription has to take into account the effectiveness of a particular pharmaceutical product in performing its intended function, such as detecting pathogens or alleviating pain. Clinical studies of health care interventions are designed and conducted to detect very modest incremental improvements. Part of the reason is that the effects of substances designed for certain functions are rarely obvious and easily detected (Glasziou, Chalmers, Rawlings, and McCulloch 2007). This makes it difficult to obtain market approval for new drugs without evidential support from clinical trials (FDA 2005).

Based on the above considerations, a problem for the ICE theory of evidence is that it is based on a misconstrued view of the evidential procedures in medicine that warrant assigning functions to medical products relative to a use plan constructed by professional designers (e.g., drug manufacturers): 
"An example would be the pharmacologists who constructed the new use plan for Aspirin that has the goal of preventing blood clots in cardiac patients. These researchers can ascribe to Aspirin the function to acetylate the enzyme cyclooxygenase in thrombocytes relative to this use plan, because they believe that Aspirin has this biochemical capacity (Bcap) and that this capacity contributes to preventing blood clots (Bcon). Moreover, these researchers can explain their effectiveness belief for the new use plan of Aspirin through an account A that consists of scientific and (bio-)technological knowledge, and that explains their beliefs Bcap and Bcon. Hence, this function ascription also satisfies our definition of function ascriptions by designers and justifiers" (Houkes et al. 2011, p. 99).

Houkes and Vermaas then go on to conclude on the same page: "Function ascriptions by professional designers, who construct new use plans for newly produced artefacts on the basis of expert knowledge, satisfy this definition in an analogous way: for an illustration, just replace the Aspirin in the last example with a hypothetical, newly developed drug that has the same capacity and a similar use plan" (p. 99).

Yet, describing how well an artifact is able to function is a methodological feat that provides evidence of a different kind to that provided in, for example, the form of an expert judgment. Chemical substances can be synthesized with the intention of achieving a desired pharmaceutical effect. How well the substance is able to perform the claimed function is, however, arguably conditioned on its (1) level of effectiveness or efficacy for a health care purpose, and (2) not harming the patient. It would be extraordinary if the justification for such a purpose had bypassed clinical trials and was instead derived from the presumed mechanism of action invoked in designing the substance, or inferred from the manufacturers use plan.

How well a medical artifact functions is typically determined by evidence from comparative clinical studies measuring the effects of its use in actual health care settings. This type of information cannot be obtained through testimony reflecting the estimates or beliefs of efficiency from experts or manufacturers, such as how he or she designed the technology. Moreover, there is no systematic way of handling (e.g., synthesizing) information from expert testimony and physiochemical reasoning about medical functions. In clinical research, a meta-analysis in a systematic review provides a very useful technique to combine and analyze information from several high-quality studies, and it is, therefore, likely to provide more definitive conclusions than the individual studies alone (Mebius 2014).

So, the problem with ICE view of evidence, which is also related to the prominent role it gives to mechanistic reasoning and testimony, is that it is unnecessarily restricted in distinguishing relative performances of the same functions. For example, a viral test with a success rate of 97 out of 100 cases will fulfill the same capacity (i.e., function) as a test that identifies 1 out of 100 . Notably, the theory of evidence employed by the ICE theorist leaves the conditions under which technologies are sufficiently effective to properly perform their designated function inadequately specified. The ICE theory of evidence leaves undetermined the cutoff point for where an effective function lies and the procedure for establishing the comparative efficiency of other medical technologies developed for the same health care purpose. The ICE theory of evidence is, therefore, not able to tackle the problem of comparing degrees of functioning (and safety) in assessing health technologies. 
As suggested earlier, many regulatory bodies will only recognize evidence from comparative clinical studies when approving medical technological products. For example, in their guidelines for diagnostic evaluations, the European Medicines Agency (EMA) (an organization similar to the American Food and Drug Administration) state their conditions for approval of diagnostic agents as follows:

"In general, the approval of a diagnostic agent is usually based on clinical indications rather than the general properties of a specific molecule... In all cases, it is required to demonstrate adequate technical and diagnostic performance of a new diagnostic agent in relation to a standard of truth and, when appropriate, to an established comparator in the clinical context in which the diagnostic agent is to be used in well-designed superiority or non-inferiority trials." (European Medicines Agency Committee for Medical Products for Human Use (CHMP) 2009, p. 15-16)

In sum, to assign a function to a health technology, it is necessary to determine that its medical function is more clinically effective than a comparable technology. A theory of evidence for ascribing medical functions to technologies needs to recognize the evidential role of clinical studies in medical research and practice. Evaluations of benefit and harm, by properly controlled trials and other clinically relevant methods, are necessary for establishing the comparative performance of health technologies for specific treatment purposes.

\section{Conclusion}

The uncertainty accompanying technological use is a common theme in health care and biotechnological innovation. This is why health care technologies are required to demonstrate efficacy and safety to help ensure, as far as possible, that interventions that are ineffective and harmful are not mistakenly adopted in medical practice. Justifying the use of health technologies requires evidential warrants establishing (1) that the technology works, (2) that it is applicable to the target condition, (3) that its use is relatively safe, and (4) that it compares well with alternative interventions.

ICE function ascriptions do not prioritize information that is relevant to patients, clinicians, and policymakers. Moreover, it has been shown that, considering the evidential basis relied on for their justification, ICE ascriptions do not take into account the relative benefit and harm of medical technologies in ensuring safe functional performance. Another aspect that is not addressed by the theory is functionality, which pertains to the degree of function exhibited by technologies. Finally, it has been suggested that the ICE theory does not take into account possible effects of biases that make function ascriptions to medical technologies unjustified.

I have argued from the perspective of clinical research and care that inferring biotechnological functions from evidence such as testimony is unjustified. Assigning functions to health care technologies requires demonstrating adequate technological performance. More specifically, the performance of a technological agent should be validated in the relevant clinical context intended for its use and, 
whenever possible, in relation to an established comparator. Decisions in the field of health care technology need to be made with reliable evidence of effectiveness, efficiency, and safety as a basis for accepting functional ascriptions to medical technologies intended for human use.

Open Access This article is distributed under the terms of the Creative Commons Attribution 4.0 International License (http://creativecommons.org/licenses/by/4.0/), which permits unrestricted use, distribution, and reproduction in any medium, provided you give appropriate credit to the original author(s) and the source, provide a link to the Creative Commons license, and indicate if changes were made.

\section{References}

Antithrombotic Trialists' (ATT) Collaboration. (2009). Aspirin in the primary and secondary prevention of vascular disease: Collaborative meta-analysis of individual participant data from randomised trials. The Lancet, 373, 1849-1860.

Ashcroft, R. (2002). What is clinical effectiveness? Studies in History and Philosophy of Science, 33, $219-233$.

FDA (2005). Part 314: Applications for FDA approval to market a new drug. Silver Spring: United States Food and Drug Administration.

Baratz, S. R., \& Goodman, C. (Eds.). (1990). Improving consensus development for health technology assessment: An international perspective. Council on Health Care Technology, Institute of Medicine. Washington, D.C.: National Academy Press.

Bijker, W. E. (1987). The social construction of Bakelite: Towards a theory of invention. In W. E. Bijker, T. P. Hughes, \& T. J. Pinch (Eds.), The social construction of technological systems: New directions in the sociology and history of technology (pp. 159-187). Cambridge: MIT Press.

Buchbinder, R., Osborne, R. H., Ebeling, P. R., Wark, J. D., Mitchell, P., Wriedt, C., et al. (2009). A randomized trial of vertebroplasty for painful osteoporotic vertebral fractures. The New England Journal of Medicine, 361, 557-568.

Buchan, W., King, M., \& Winston, R. (2012). Can onions cure ear-ache? Oxford: Bodleian Library Publishing. Bunge, M. (1985). Treatise on basic philosophy (Vol. 7, Part II). Dordrecht: Reidel.

Canadian Task Force on Preventive Health Care. CTFPHC History/Methodology. http://canadiantaskforce. ca/ctfphc-guidelines/overview. Accessed 04 July 2016.

Cartwright, N., \& Hardie, J. (2012). Evidence based policy: A practical guide to doing it better. Oxford: Oxford University Press.

Chalmers, I., \& Glasziou, P. (2009). Avoidable waste in the production and reporting of research evidence. The Lancet, 374, 86-89.

Chen, J. F., Wu, C. T., \& Lee, S. T. (2004). Percutaneous vertebroplasty for the treatment of burst fractures. Case report. Journal of Neurosurgery: Spine, 1, 228-231.

European Medicines Agency Committee for Medical Products for Human Use (CHMP) (2009). Guideline on clinical evaluation of diagnostic agents. http://www.ema.europa.eu/ema/pages/includes/document/open document.jsp?webContentId=WC500003584 (Accessed 04 July 2016).

Evans, I., Thornton, H., Chalmers, I., \& Glasziou, P. (2010). Testing treatments. Better research for better healthcare (2nd ed.). London: Pinter \& Martin.

Evidence-Based Medicine Working Group. (1992). Evidence-based medicine. A new approach to teaching the practice of medicine. Journal of the American Medical Association, 268, 2420-2425.

Fransen, M., \& McConnell, S. (2008). Exercise for osteoarthritis of the knee. Cochrane Database of Systematic Reviews, 4(4), CD004376.

Glasziou, P., Chalmers, I., Rawlings, M., \& McCulloch, P. (2007). When are randomised trials unnecessary? Picking signal from noise. British Medical Journal, 334, 349-351.

Gilbert, R., Salanti, G., Harden, M., \& See, S. (2005). Infant sleeping position and the sudden infant death syndrome: Systematic review of observational studies and historical review of clinicians? Recommendations from 1940-2000. International Journal of Epidemiology, 34, 74-87.

Guyatt, G. H., et al. (2008). GRADE: An emerging consensus on rating quality of evidence and strength of recommendations. British Medical Journal, 336, 924-926.

Hansson, S. O. (2006). Defining technical function. Studies in History and Philosophy of Science, 37, 19-22. 
Hansson, S. O. (2014). Why and for what are clinical trials the gold standard? Scandinavian Journal of Public Health, 42, 41-48.

Hansson, S. O. (2015). Experiments before science. What science learned from technological experiments. In S. O. Hansson (Ed.), The role of technology in science: philosophical perspectives (pp. 81-110). Dordrecht: Springer Media.

Higgins, J.P.T., \& Green, S. (Eds.), (2011). Cochrane handbook for systematic reviews of interventions. Version 5.1.0 [updated March 2011]. The Cochrane Collaboration. www.cochrane-handbook.org.

Houkes, W. (2006). Knowledge of artefact functions. Studies in History and Philosophy of Science, 37, $102-113$.

Houkes, W., Kroes, P., Meijers, A., \& Vermaas, P. E. (2011). Dual-nature and collectivist frameworks for technical artifacts: a constructive comparison. Studies in History and Philosophy of Science, 42, 198-205.

Houkes, W., \& Vermaas, P. E. (2010). Technical functions: On the use and design of artefacts. Dordrecht: Springer.

Howick, J. (2011). The philosophy of evidence-based medicine. Chichester: Blackwell-Wiley.

Howick, J. (2016). Justification of Evidence-Based Medicine Epistemology. The Bloomsbury Companion to Contemporary Philosophy of Medicine 113.

Howick, J., Kennedy, A. G., \& Mebius, A. (2015). Philosophy of evidence-based medicine. In D. Pritchard (Ed.), Oxford bibliographies in philosophy. New York: Oxford University Press.

Howick, J. \& Mebius, A. (2014). In search of justification for the unpredictability paradox. Trials, 15, 480.

Health Technology Assessment (HTA) (2014) . http://www.nets.nihr.ac.uk/programmes/hta/remit.

Jensen, M. E., Evans, A. J., Mathis, J. M., Kallmes, D. F., Cloft, H. J., \& Dion, J. E. (1997). Percutaneous polymethylmethacrylate vertebroplasty in the treatment of osteoporotic vertebral body compression fractures: technical aspects. American Journal of Neuroradiology, 18, 1897-1904.

Kallmes, D. F., Comstock, B. A., Heagerty, P. J., Turner, J. A., Wilson, D. J., Diamond, T. H., et al. (2009). A randomized trial of vertebroplasty for osteoporotic spinal fractures. New England Journal of Medicine, $361,569-579$.

Kennedy, A. G. (2013). Differential diagnosis and the suspension of judgment. Journal of Medicine and Philosophy, 38, 487-500.

Knottnerus, J. A., Buntinx, F., \& van Weel, C. (2009). General introduction: evaluation of diagnostic procedures. Oxford: Wiley-Blackwell. doi:10.1002/9781444300574.ch1

Kroes, P. (2002). Designing methodology and the nature of technical artefacts. Design Studies, 23, $287-302$.

La Caze, A., \& Colyvan, M. (2016). A challenge for evidence-based policy (Axiomathes, pp. 1-13).

Marghoob, A. A., Changchien, L., DeFazio, J., Dessio, W. C., Malvehy, J., Zalaudek, I., et al. (2009). The most common challenges in melanoma diagnosis and how to avoid them. Australian Journal of Dermatology, 50, 1-15.

McCloskey, R. V., Straube, R. C., Sanders, C., Smith, S. M., \& Smith, C. R. (1994). Treatment of septic shock with human monoclonal antibody HA-1A: A randomized, double-blind, placebo-controlled trial. CHESS Trial Study Group. Annals of Internal Medicine, 121, 1-5.

Mebius, A., Kennedy, A. G., \& Howick, J. (2016). Research gaps in the philosophy of evidence-based medicine. Philosophy Compass 11/11, 757-771. doi:10.1111/phc3.12352

Mebius, A. (2015). Philosophical controversies in the evaluation of medical treatments: with a Focus on the evidential roles of randomization and mechanisms in evidence-based medicine. Theses in philosophy from the Royal Institute of Technology, ISSN 1650-8831.

Mebius, A. (2014). A weakened mechanism is still a mechanism: On the causal role of absences in mechanistic explanation. Studies in History and Philosophy of Biological and Biomedical Sciences, 45, 43-48.

Moore, T. (1995). Deadly medicine. New York: Simon \& Schuster.

Morton, C. A., \& Mackie, R. M. (1998). Clinical accuracy of the diagnosis of cutaneous malignant melanoma. British Journal of Dermatology, 138, 283-287.

Nordström, P. (2011). Technology education and non-scientific technological knowledge (Theses in philosophy from the Royal Institute of Technology, ISSN 1650-8831).

OCEBM Levels of Evidence Working Group (2011). The Oxford levels of evidence 2. Oxford Centre for Evidence-Based Medicine. http://www.cebm.net/ocebm-levels-of-evidence/. Accessed 04 July 2016.

Parvizi, N., \& Woods, K. (2014). Regulation of medicines and medical devices: Contrasts and similarities. Clinical Medicine, 14, 6-12.

Pound, P., \& Bliss, S. D. (2014). Is animal research sufficiently evidence based to be a cornerstone of biomedical research? British Medical Journal, 348, g3387.

Prasad, V., \& Ho, N. (2014). Why do we continue to adopt medical practices based on pathophysiology alone, when we should be insisting on clinical trials. Journal of Clinical Epidemiology, 67, 361-363.

Prasad, V., Ho, N., \& Ioannidis, J. P. A. (2014). Evidence-based de-implementation for contradicted, unproven, and aspiring healthcare practices. Implementation Science, 9, 1.

Sackett, D. L. (1996). Evidence based medicine: what it is and what it isn't. British Medical Journal, 13, 71-72. 
Sackett, D. L., et al. (1999). Time to put the Canadian Institutes of Health Research on trial. Canadian Medical Association Journal, 161, 1414-1415.

Schulz, K. F., Chalmers, I., Hayes, R. J., \& Altman, D. G. (1995). Empirical evidence of bias. Dimensions of methodological quality associated with estimates of treatment effects in controlled trials. JAMA 273, 408-412

Schünemann, H., Oxman, A. D., Brozek, J., Glasziou, P., Jaeschke, R., Vist, G. E., et al. (2008). Grading quality of evidence and strength of recommendations for diagnostic tests and strategies. British Medical Journal, 336, 1106-1110.

Shea, B., Grimshaw, J., Wells, G., Boers, M., Andersson, N., Hamel, C., et al. (2007). Development of AMSTAR: A measurement tool to assess the methodological quality of systematic reviews. BMC Medical Research Methodology, 7, 10.

Smith, T. (1921). Extracts from the diary of a huntsman. London: E. Arnold \& co.

Straus, S., Glasziou, P., Richardson, W. S., \& Haynes, B. (2011). Evidence-based medicine: How to practice and teach it (4th ed.). Edinburgh: Churchill Livingstone.

Tröhler, U. (2012). To improve the evidence of medicine: The 18th century origins of a critical approach. Edinburg: Royal College of Physicians.

Vermaas, P. E., \& Houkes, W. (2003). Ascribing functions to technical artefacts: A challenge to etiological accounts of functions. British Journal for the Philosophy of Science, 54, 261-289.

Vermaas, P. E., \& Houkes, W. (2006). Technical functions: A drawbridge between the intentional and structural natures of technical artefacts. Studies in History and Philosophy of Science, 37(1), 5-18.

Vodopivec-Jamsek, V., de Jongh, T., Gurol-Uranci, I., Atun, R., \& Car, J. (2012). Mobile phone messaging for preventive health care. Cochrane Database of Systematic Reviews, 12, CD007457.

Worrall, J. (2002). What evidence in evidence-based medicine? Philosophy of Science, 69, S316-S330. 